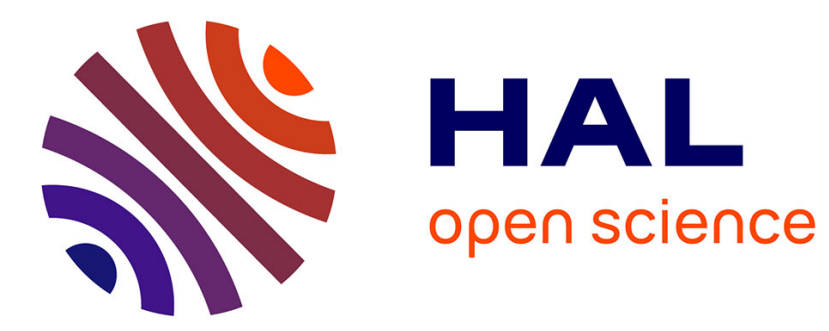

\title{
Bulk Photorefractive Semiconductors
}

Philippe Delaye, H.J. von Bardeleben, Gérald Roosen

\section{To cite this version:}

Philippe Delaye, H.J. von Bardeleben, Gérald Roosen. Bulk Photorefractive Semiconductors. MRS

Bulletin, 1994, Mars, pp.39. hal-00677576

\section{HAL Id: hal-00677576 \\ https://hal-iogs.archives-ouvertes.fr/hal-00677576}

Submitted on 30 Mar 2012

HAL is a multi-disciplinary open access archive for the deposit and dissemination of scientific research documents, whether they are published or not. The documents may come from teaching and research institutions in France or abroad, or from public or private research centers.
L'archive ouverte pluridisciplinaire HAL, est destinée au dépôt et à la diffusion de documents scientifiques de niveau recherche, publiés ou non, émanant des établissements d'enseignement et de recherche français ou étrangers, des laboratoires publics ou privés. 


\title{
BULK PHOTOREFRACTIVE SEMICONDUCTORS
}

\author{
Ph. Delaye(1), H.J. Von Bardeleben(2), G. Roosen(1) \\ (1) Institut d'Optique Théorique et Appliquée, Unité de Recherche Associée \\ 14, CNRS, Bât.503 - Centre Universitaire d'Orsay, B.P. 147 - 91403 \\ ORSAY Cedex \\ (2) Groupe de Physique du Solide, Unité de Recherche Associée 17, CNRS \\ Université Paris 6 et Paris 7 - 2, Place de Jussieu - 75005 PARIS
}

The photorefractive effect (PRE) has been studied for more than twenty five years now 1,2 and many applications for optical signal processing such as correlation, real time holography, dynamic interconnections and optical memories have been developed. The materials, in which the PRE has been studied, were mainly oxides (ferroelectrics and sillenites), in which the useful spectral range lies in the visible. Applications for telecommunication systems and eye-safe devices have required an extension of the spectral range into the near infrared $(1.0$ to $1.5 \mu \mathrm{m})$ and thus different materials have to be explored. It has been shown that the bulk semiinsulating III-V semiconductors GaAs and $\mathrm{InP}^{3}$ and more recently the II-VI compound CdTe ${ }^{4}$ were efficient materials for this spectral range. III-V materials offer the great advantage of being available as bulk semiinsulating materials of high crystalline perfection and homogeneity as regards to their electrical properties due to their importance as substrate materials in micro and optoelectronic technology. However these materials were not optimized for PRE applications and thus quantitative analyses of PRE experiments 5 related to the specific material defect properties are 
necessary for further developments. It has equally been shown, that the PRE can be used as an efficient tool for the material characterization 6,7 .

\section{THE PHOTOREFRACTIVE EFFECT IN BULK SEMICONDUCTORS}

The PRE in a particular material depends on both its extrinsic, defect related and intrinsic properties : photoconductivity and carrier diffusion properties on one side and the electro-optic coefficients, dielectric constants and refractive index on the other. Photoconductivity is responsible for the creation of a space-charge field by a spatial redistribution of the trapped charges in the deep level due to a non uniform illumination by photons of below band gap energy. The free carriers, created by photoionization of deep levels in the bright region of the illumination pattern, take part in the redistribution of the charges through diffusion (no external applied field) or drift (under external applied field) towards dark region where they recombine. The electrooptic effect transforms the space-charge field in a spatially modulated index variation.

These basic mechanisms give hints of the key properties of the PRE :

1- The strength of the space-charge field depends on the quantity of charges redistributed and then is linked to the concentration of the deep level and to the defect occupancy.

2- The number of redistributed charges directly depends on the number of photons absorbed in the material, making the PRE strength governed by the optical beam energy (note that its speed is beam power dependent). Thus noticeable refractive index modulations are obtained with low power CW lasers.

3- The strength of the space-charge field can also be increased by applying an electric field leading to a charge redistribution effect controlled by the drift of free carriers, that is more efficient than their diffusion. 
The expression of the photoinduced space-charge field is derived following the band transport model 8,9 with a single defect in a concentration $\mathrm{N}_{\mathrm{T}}$ which ionized part is in concentration $\mathrm{N}_{0}$ and neutral part in concentration $\mathrm{N}_{\mathrm{T}}-\mathrm{N}_{0}$ (Fig.1). We consider both thermal (ß) and optical (S) emission of electrons and holes from the defect. The illumination is given by a spatially modulated interference pattern along coordinate axis $\mathrm{z}$, $I=I_{0}\left(1+\operatorname{Re}\left(m e^{i k z}\right)\right)$ with a wave number $\mathrm{k}\left(k=\frac{2 \pi}{\Lambda}, \Lambda=\frac{\lambda}{2 n_{0} \sin \theta}\right.$ grating spacing with $\theta$ the half-angle between the two interfering beams inside the crystal, $\mathrm{n}_{0}$ is the linear refractive index of the material, and $\lambda$ the wavelength), a modulation $\mathrm{m}$ and a total incident illumination $\mathrm{I}_{0}$. In first approximation the space-charge field is spatially modulated with the same grating spacing than the illumination pattern and, when the stationary regime is reached, its amplitude is (for grating spacing smaller than the diffusion length of the carriers and no applied electric field) :

$$
E_{1}=-i m \frac{k_{B} T}{e} \frac{k}{1+\frac{k^{2}}{k_{0}^{2}}} \xi_{0}(1)
$$

$\xi_{0}=\frac{S_{n}\left(N_{T}-N_{0}\right)-S_{p} N_{0}}{A_{n} S_{n}\left(N_{T}-N_{0}\right)+A_{p} S_{p} N_{0}}$ is the electron-hole competition coefficient, with $A_{n}=\frac{\beta_{n}+S_{n} I_{0}}{S_{n} I_{0}}$ and $A_{p}=\frac{\beta_{p}+S_{p} I_{0}}{S_{p} I_{0}}, \frac{k_{B} T}{e} \approx 0.025 \mathrm{eV}$.

$\alpha_{n}=S_{n}\left(N_{T}-N_{0}\right)$ (resp. $\left.\alpha_{p}=S_{p} N_{0}\right)$ corresponds to the part of absorption that generates electrons (resp. holes). Note that for generation of electrons only (or holes only) $\xi_{0}$ is maximal $(\approx \pm 1)$.

$k_{0}^{2}=\frac{e^{2}}{\varepsilon k_{B} T} N_{e f f}$ is the inverse squared of the Debye screening length with the effective trap density $N_{\text {eff }}=\frac{N_{0}\left(N_{T}-N_{0}\right)}{N_{T}}$ ( $\varepsilon$ is the dielectric constant).

$E_{1}$ is purely imaginary, which means that the space-charge field grating is $\pi / 2$-shifted from the illumination grating.

Through the linear electrooptic effect (Pockels effect), the spacecharge field grating gives rise to an index grating of amplitude 10 : 


$$
\Delta n=\frac{n_{0}^{3} r_{e f f} E_{1}}{2}
$$

$r_{\text {eff }}$ is the effective electrooptic coefficient which depends on the orientation of the sample and on polarization of beams ${ }^{11}$. For example in the classical beam-coupling configuration, i.e. grating vector along [001], propagation along [ $\overline{1} 10]$ and beam polarization along [110], for crystal of the $\overline{4} 3 \mathrm{~m}$ class of symmetry such as GaAs, InP or CdTe, we have $r_{\text {eff }}=r_{41}$.

The diffraction of the two beams on the index grating they create, gives an energy transfer from one beam to the other one ${ }^{8}$. This two wave mixing (TWM) process is characterized by a photorefractive gain $\Gamma$ :

$$
\Gamma=\frac{2 \pi n_{0}^{3} r_{41}}{\lambda \cos \theta} \frac{k_{B} T}{e} \frac{k}{1+\frac{k^{2}}{k_{0}^{2}}} \xi_{0}(3)
$$

Experimentally the energy transfer is measured as the ratio $\gamma_{0}$ of the intensity of a probe beam with and without the presence of a coherent pump beam (Fig.2). In the condition of high pump-to-probe beam ratio (i.e. small modulation of the fringes), $\gamma_{0}$ is written :

$$
\gamma_{0}=e^{\Gamma l}(4)
$$

\section{TWO BEAM COUPLING IN SEMIINSULATING GaAs : A QUANTITATIVE EXAMPLE}

We now present, as an illustration, photorefractive experiments performed on a well characterized semiconductor : semiinsulating undoped GaAs. The PRE is analyzed through a TWM experiment in which we measure the energy transfer from a pump beam to a probe beam as a function of the grating wavenumber $\mathrm{k}$. This is achieved at the two wavelengths $1.06 \mu \mathrm{m}$ and $1.32 \mu \mathrm{m}$ given by $\mathrm{CW}$ diode-pumped Nd-YAG lasers. From the measurement of the effective gain $\gamma_{0}$ (Fig.2), we deduce 
photorefractive gain $\Gamma$ (Fig.3) according to expression (4). The results are analyzed using equation (3), in which the extrinsic material properties are contained in the electron-hole competition coefficient $\xi_{0}$ and $\mathrm{N}_{\mathrm{eff}}$ the deep level effective trap density (i.e. $k_{0}^{2}$ ).

In LEC grown undoped GaAs the semiinsulating character of the material is due to the compensation of the residual shallow donors and acceptors, with concentrations $\mathrm{N}_{\mathrm{A}}>\mathrm{N}_{\mathrm{D}} \approx 10^{15} \mathrm{~cm}^{-3}$, by the intrinsic EL2 defect $\left(\mathrm{N}_{\mathrm{EL} 2} \approx 1 \ldots .2 \times 10^{16} \mathrm{~cm}^{-3}\right)^{12}$, which gives rise to a deep $0 /+$ level at $0.75 \mathrm{eV}$ below the bottom of the conduction band ${ }^{13}$. EL2 defect is an arsenic-antisite related double donor defect. The electrical compensation and, related with this, the atomic structure of the EL2 defect have been the object of numerous studies and recent reviews are available ${ }^{14}$. Whereas the total EL2 concentration is relatively constant, around $2 \times 10^{16} \mathrm{~cm}^{-3}$ for high pressure LEC grown materials, the occupation ratio $\left[E L 2^{+}\right] /\left[E L 2^{0}\right]$ depends on the crystal growth conditions and on parameters such as the purity of the starting materials or the humidity of the encapsulation but equally on the position in the ingot. Occupation ratio between 1 and 0.1 can be found, but modern state of the art material optimized as substrate materials for microelectronics applications is characterized by a value of about 0.1. The EL2 defect, which is at the origin of the electrical compensation, is equally responsible for the photoconductivity in the $1-1.5 \mu \mathrm{m}$ spectral range 15 due to the optical cross sections $S_{n}$ and $S_{p}$ of $E L 2^{\circ}$ and $E L 2^{+}$respectively, which are both of importance at these excitation energies. The determination of the absolute values of these cross sections at room temperature has only been achieved recently 16 .

The quantitative analysis of the TWM experiment requires further the knowledge of the absolute concentrations of $E L 2^{\circ}$ and $E L 2^{+}$; the values can either be obtained by an all optical absorption measurement at two different wavelengths 17 or by the application of the electron paramagnetic 
resonance (EPR) technique concerning $E L 2^{+} 18$. The application of this last technique is generally preferable due to its defect selective character, whereas the quality of an absorption measurement depends strongly on the absence of other deep levels. The EPR spectrum (Fig.4) of the $E L 2^{+}$defect is described by the Spin Hamiltonian :

$$
\mathrm{H}=\mathrm{g} \beta \mathrm{BS}+\mathrm{AIS}(5)
$$

with the Landé factor $\mathrm{g}=2.04$ and the central hyperfine interaction constant $\mathrm{A}=890 \times 10^{-4} \mathrm{~cm}^{-1}$, both isotropic (with $B$ the Bohr magneton and $\mathrm{B}$ the magnetic field, $S=1 / 2$ is the electronic spin and $I=3 / 2$ is the nuclear spin) . The spin concentration is obtained by a double integration of the experimental first derivative spectrum and a comparison with a spin standard sample.

With the help of the concentrations determined in the sample from absorption measurement for $\left\lfloor E L 2^{\circ}\right\rfloor$ and from EPR measurements for $\left[E L 2^{+}\right]$, as well as the other parameters taken from the literature $16,19,20$ we calculate the photorefractive gain (Fig.3). We find a good agreement between calculated values and the experimental ones, thus confirming the model for the photorefractive effect. The change of the sign of photorefractive gain from $\lambda=1.06 \mu \mathrm{m}$ to $\lambda=1.32 \mu \mathrm{m}$, indicates a change of the majority carrier for PRE. These curves have been obtained for a GaAs sample in which the compensation ratio $\left[E L 2^{+}\right] /\left[E L 2^{0}\right]$ is close to one. Thus the sign reversal for $\xi_{0}$ follows the variation of the $S_{n} / S_{p}$ ratio with wavelength $\left(\mathrm{S}_{\mathrm{n}} / \mathrm{S}_{\mathrm{p}}>1\right.$ for $\lambda=1.06 \mu \mathrm{m} ; \mathrm{S}_{\mathrm{n}} / \mathrm{S}_{\mathrm{p}}>$ for $\left.\lambda=1.32 \mu \mathrm{m}^{16}\right)$.

The importance of the electron-hole competition in PRE experiments is well illustrated by these results : in particular at the wavelength of $1.32 \mu \mathrm{m}$ the electron-hole competition is very sensitive to the compensation ratio due to the increasing importance of $S_{p}$ over $S_{n}$. Thus a sample with a ratio $\left[E L 2^{+}\right] /\left[E L 2^{0}\right]$ close to 0.1 will not present a reverse of the sign of 
the gain between $1.06 \mu \mathrm{m}$ and $1.32 \mu \mathrm{m}$ contrary to this sample, this is what we indeed observed in our experiments. It becomes further clear that materials optimized for applications at $1.06 \mu \mathrm{m}$ are not necessarily suited for applications at $1.5 \mu \mathrm{m}$. The optimization of the performances of GaAs at different wavelengths thus requires materials with different compensation ratios ; these can in principle be adapted by the growth conditions, but as currently semiinsulating GaAs is grown for microelectronics applications such material is not available on the market. A second approach would be to modify the compensation by post growth treatments such as electron irradiation or thermal treatments 21,22 .

\section{A COMPARISON OF INFRARED ACTIVE MATERIALS}

The evaluation of photorefractive performances of a material can be assessed through different criteria, such as the required irradiance, the electron-hole competition rate, the effective trap density or the refractive index change. We will now precise all these points.

In insulating materials, we usually consider that the steady-state value of the PRE does not depend on the incident illumination, which only affects the time constant at which the steady-state is reached. In semiconductors, this is a little more complex due to the semiinsulating nature of the material. Carriers can be excited from the deep level either optically or thermally. The thermal emission is not spatially modulated and its effect will be an isotropic redistribution of charges that will decrease the strength of the space charge field. This influence is expressed by the terms $A_{n}$ and $A_{p}$ in the $\xi_{0}$ expression (1). In a one carrier model (for example electrons), the photorefractive gain can be rewritten : 


$$
\Gamma=\frac{\Gamma_{\infty}}{1+\frac{\beta_{n}}{S_{n} I_{0}}}(6)
$$

$\Gamma_{\infty}$ is the photorefractive gain obtained at an irradiance giving a number of photoexcited carriers larger than the thermally excited one $: S_{n} I_{0}>\beta_{n}$, i.e. $A_{n}=1$. At low irradiance the gain is reduced due to the influence of $\beta_{n}$. We define the equivalent dark irradiance $I_{d}$ as the irradiance for which the gain is reduced by a factor 2 . It corresponds, in a one defect model, to : $S_{n} I_{d}=\beta_{n}$. In semiconductors with a monopolar conduction, the conductivity is defined as :

$$
\sigma=\sigma_{d}+\sigma_{p h}=\frac{e \mu_{n} \beta_{n}\left(N_{T}-N_{0}\right)}{\gamma_{n} N_{0}}+\frac{e \mu_{n} S_{n}\left(N_{T}-N_{0}\right)}{\gamma_{n} N_{0}} I_{0} \text { (7) }
$$

where $\mu_{\mathrm{n}}\left(\mu_{\mathrm{p}}\right)$ is the electron (hole) mobility, and $\gamma_{\mathrm{n}}\left(\gamma_{\mathrm{p}}\right)$ the recombination coefficient of electrons (holes). This means that $I_{d}$ can be defined as the irradiance for which photoconductivity $\sigma_{\mathrm{ph}}$ equals dark conductivity $\sigma_{\mathrm{d}}$. In a bipolar model, $\mathrm{I}_{\mathrm{d}}$ can always be defined but its relation to conductivity is not as simple. Nevertheless, the comparison of photoconductivity and dark conductivity gives a good clue to the illumination dependence of photorefractive gain. We thus have a first criteria for the performance of a photorefractive crystal : the photoconductivity must overpass the dark conductivity. This condition gives the minimal irradiance at which a crystal can be effectively used (i.e. $A_{n}=A_{p}=1$ ).

The second important point is the electron-hole competition factor :

$$
\xi_{0}=\frac{S_{n}\left(N_{T}-N_{0}\right)-S_{p} N_{0}}{S_{n}\left(N_{T}-N_{0}\right)+S_{p} N_{0}}
$$

This coefficient varies between -1 and 1 , the optimum condition being $\left|\xi_{0}\right|=1$, i.e. monopolar case. The sign of $\xi_{0}$ gives the majority carrier for PRE : electrons correspond to $\xi_{0}>0$, (holes to $\xi_{0}<0$ ). In the case of an exact compensation (i.e. $\mathrm{S}_{\mathrm{n}}\left(\mathrm{N}_{\mathrm{T}}-\mathrm{N}_{0}\right)=\mathrm{S}_{\mathrm{p}} \mathrm{N}_{0}$ ), the photorefractive gain equals zero. A good photorefractive crystal should be the closest as possible to the monopolar case in order to exhibit high gains. This parameter is often hard 
to modify by post growth treatments as discussed above on the example of GaAs. For a fixed wavelength and a given defect only a modification in the compensation ratio $\left(\mathrm{N}_{\mathrm{T}}-\mathrm{N}_{0}\right) / \mathrm{N}_{0}$ can help to change the electron-hole competition. This implies a good control of the Fermi level in the as-grown crystals. As the optical cross sections $S_{n}$ and $S_{p}$ depend on the photon energy we have for each case an optimum wavelength ; however for practical reasons the wavelengths are fixed and this parameter can not be varied freely. Different doping can equally help to change the range of utilization of a given material. In all cases it is necessary to characterize quantitatively the defect relevant for the PRE.

The third criteria deals with the effective trap density $\mathrm{N}_{\text {eff }}$. This quantity gives the maximum number of charges that can be redistributed and then the maximum space-charge field that can be induced. For a defect under two states of charge, $\mathrm{N}_{\mathrm{eff}}$ is given by the minority species, which means that even if a high defect concentration is obtainable, care as to be taken in the control of the compensation ratio of the material. Note that an increase of the concentration of the defect (by higher doping for example) also increases $\alpha$ the absorption of the crystal.

The last point to fully characterize the induced index change is given by the parameter $n_{0}^{3} r_{41}$. The higher $n_{0}^{3} r_{41}$, the higher the photorefractive gain. This gives for example an argument in favor of II-VI semiconductors for which $n_{0}^{3} r_{41}$ is twice higher than for III-V materials.

Three materials are widely used for PRE in the infrared : undoped GaAs 3,5,23, Iron doped InP 3,24,25 and more recently Vanadium doped CdTe 4,26,27. Their characteristics are presented in Table1. Some other semiconductors are also studied $\left(\mathrm{GaP}^{33}, \mathrm{ZnTe}^{34}, \mathrm{CdSe}^{35}\right)$ but they are mainly developed for their properties in the visible even if some (ZnTe) have sensitivity which extends towards $1.32 \mu \mathrm{m}$. Another possibility is the 
extension to alloys ${ }^{36}$, such as CdZnTe or CdMnTe which allow a choice of the band gap value and then a possible adjustment of some of their properties.

If we look at Table 1, keeping in mind the four important criteria for performances previously defined, we see that CdTe has the higher index change due to its higher electrooptic coefficient. Its sensitivity range extends beyond $1.5 \mu \mathrm{m}$. GaAs is the crystal that possess the highest photoconductivity but, as discussed previously, the important point is the ratio between dark conductivity and photoconductivity. According to this criteria CdTe can be used with lower irradiance (of the order of $\left.0.1 \mathrm{~mW} \cdot \mathrm{cm}^{-2}\right)$. Working with extrinsic semiconductors, the dark conductivity is related to the defect, giving rise to the Fermi level pinning. This Fermi level pinning can lead to dark conductivity close to the intrinsic conductivity, determined by the energy band gap of the material. For example, undoped Bridgman CdTe is n-type conductive and then unsuitable for photorefractive applications; it is the vanadium doping which leads via a deep $\mathrm{V}^{0 /+}$ donor level and non identified acceptors to high resistive and photoconductive material ${ }^{37}$.

The highest photorefractive gain without applied electric field is obviously obtained in CdTe due to its higher electrooptic coefficient. When considering results currently obtained under applied DC field, the highest gains were obtained in InP:Fe. Indeed a resonant enhancement is achieved when photogeneration of holes and thermal emission of electrons equal each other 25 . This technique, only demonstrated for InP:Fe, requires an accurate control of both illumination and temperature. Classical techniques use either DC field and moving gratings 23 or AC field. This last techniques are currently developped in CdTe to obtain high photorefractive gains 27,32. Application of high electric fields raise equally the problem of suitable electrical contacts, homogeneity of the field distribution inside the crystal 
and the fact that defect properties can be sensitive to the electric field strength. This last point for example is of importance for the EL2 defect in GaAs 38 . The field dependent capture cross section of EL2 39 leads, for fields above $300 \mathrm{~V} . \mathrm{cm}^{-1}$, to the formation of moving high field domains. These problems may impede the use of GaAs in applications requiring external applied fields.

According to the values in Table 1 , CdTe seems to be the most promising crystal for use in the $1.3-1.5 \mu \mathrm{m}$ spectral range with low power laser diodes. However the defect properties of CdTe:V are much less known than those of III-V semiconductors and more studies are required and are currently under way in various laboratories in order to optimize CdTe: $\mathrm{V}$ for PRE applications.

\section{CONCLUSION}

The main particularity of the photorefractive effect, that makes it attractive for beam processing, is an optical nonlinearity leading to efficient energy transfer and phase conjugation with low power lasers. The materials that are sensitive in the near infrared are the semiinsulating semiconductors. Good correlation is obtained between the theoretical model describing the PRE and experimental results in that kind of crystals. This points out a possible use of the PRE as a characterization technique of deep levels in these materials which are of importance as substrates for microelectronics. For optical processing applications in the near infrared, optimization has still to be continued in order to obtain an optimum effect for specified wavelengths. Nevertheless the magnitude of the effects already obtained has lead to the beginning of device realizations in different research groups.

An example of such a device is the Double Phase Conjugate Mirror (DPCM) ${ }^{40}$. The DPCM allows to couple two mutually incoherent beams 
and to create their phase conjugates. The great interest of such a device is that the temporal information of one beam is transferred to the phase conjugate of the other beam. Consequently, a DPCM can realize a wavefront conversion of a beam having an arbitrary wavefront into a beam with a perfect wavefront. That makes this device important for beam processing in free space telecommunication systems. High gain are necessary to overpass the threshold of devices like DPCM $((\Gamma-\alpha) \ell>4)$, which means that we are in search for a crystal that presents high photorefractive gain in the spectral range $1.3-1.5 \mu \mathrm{m}$ with the low energies given by laser diodes 


\section{REFERENCES :}

[1] A.Ashkin, G.D.Boyd, J.M.Dziedzic, R.G.Smith, A.A.Ballman, H.J.Levinstein, K.Nassau, Appl. Phys. Lett. 9 (1966) p.72

[2] P.Günter, J.P.Huignard, Photorefractive materials and their applications I and II Vol 61-62 ( Springer Verlag, 1988).

[3] A.M.Glass, A.M.Johnson, D.H.Olson, W.Simpson, A.A.Ballman, Appl. Phys. Lett. 44 (1984) p.948

[4] R.B.Bylsma, P.M.bridenbaugh, D.H.Olson, A.M.Glass, Appl. Phys. Lett. 51 (1987) p.889

[5] M.B.Klein, Opt. Lett. 9 (1984), p.350.

[6] R.B.Bylsma, D.H.Olson, A.M.Glass, Appl. Phys. Lett. 52 (1988) p.1083

[7] K.Jarasiunas, Ph.Delaye, G.Roosen, Phys. Stat. Sol. B175 (1993) p. 445

[8] N.V. Kukhtarev, V.B. Markov, S.G. Odulov, M.S. Soskin, V.L. Vinetskii, Ferroelectrics 22 (1979) p.949; 22 (1979) p.961.

[9] F.P. Strohkendl, J.M.C. Jonathan, R.W. Hellwarth, Opt. Lett. 11 (1986), p.312.

[10] H. Kogelnik, Bell. System. Technical. Journal. 48 (1969) p.2909.

[11] J. C. Fabre, J.M.C. Jonathan, G. Roosen, Opt. Comm. 65 (1988), p.257.

[12] G.M.Martin, J.P.Farges, G.Jacob, J.P.Hallais, G.Poiblaud, J.Appl. Phys. 51 (1980) p.2840

[13] E.J. Johnson, J.A. Kafalas, R.W. Davies. J. Appl. Phys. 54 (1983), p.204.

[14] See for example : J.C.Bourgoin, H.J.Von Bardeleben, D.Stiévenard. J. Appl. Phys. 64 (1988), p.R65.

[15] M.Kaminska, Revue. Phys. Appl. 23 (1988), p.793. 
[16] P.Silverberg, P.Omling, L.Samuelson. Appl. Phys. Lett. 52 (1988), p.1689.

[17] F.X. Zach, A. Winnacker. Jap. J. Appl. Phys. 28 (1989), p.6.

[18] R.J. Wagner, J.J. Krebs, G.H. Strauss, A.M. White. Sol. State Commun. 36 (1980), p.15.

[19] D.T.F. Marple, J. Appl. Phys. 35 (1964), p.1241.

[20] C.A. Berseth, C. Wuethrich, F.K. Reinhart, J. Appl. Phys. 71 (1992), p.2821.

[21] Ph. Delaye, H.J. Von Bardeleben, G.Roosen, in Digest of Topical Meeting on Photorefractive Materials, Effects and Devices IV (6-10 august 1993, Kiev) Paper FrC02.

[22] L.J.Cheng, J.Lagowski, M.F.Rau, F.C.Wang. Radiation Effect And Defect In Solid 111 (1989), p.37

[23] B. Imbert, H. Rajbenbach, S. Mallick, J.P. Herriau, J.P. Huignard, Opt. Lett. 13 (1988) p.327.

[24] P. Delaye, P. U. Halter, G. Roosen, J. Opt Soc. Am. B 7 (1990), p.2268 .

[25] G. Picoli, P. Gravey, C. Ozkul, V. Vieux.J. Appl. Phys. 66 (1989), p.3798.

[26] J.C.Launay, V.Mazoyer, M.Tapiero, J.P.Zielinger, Z.Guellil, Ph.Delaye, G.Roosen, Appl. Phys. A 55 (1992), p.33.

[27] M.Ziari, W.H.Steier, P.M.Ranon, M.B.Klein, S.Trivedi, J.Opt.Soc.Am.B 9 (1992) p.1461.

[28] N. Suzuki, .K. Tada, Jpn. J. Appl. Phys. 23 (1984) p.291.

[29] D.T.F. Marple, J. Appl. Phys. 35 (1964), p.539.

[30] D.T.H.Liu, L.J.Cheng, M.F.Rau, F.C.Wang, Appl. Phys. Lett. 53 (1988) p.1369

[31] C.Ozkul, G.Picoli, P.Gravey, N.Wolffer, Appl. Opt. 29 (1990) p.2711. 
[32] Y.Belaud, P.Delaye, J.C.Launay, G.Roosen, accepted for publication in Opt.Commun. (1993)

[33] K. Kuroda, Y. Okazaki, T. Shimura, H.Okamura, M. Chihara, M. Itoh, I. Ogura, Opt. Lett. 15 (1990) p.1197.

[34] M.Ziari, W.H.Steier, P.M.Ranon, S.Trivedi, M.B.Klein, Appl. Phys. Lett. 60 (1992), p.1052.

[35] P.Tayebati, J. Kumar, S.Scott Appl. Phys. Lett. 59 (1991), p.3366.

[36] M.Ziari, W.H.Steier, M.B.Klein, S.Trivedi, in Digest of Topical Meeting on Photorefractive Materials, Effects and Devices III (August 1991, Beverly) Paper TuA6.

[37] H.J. Von Bardeleben, J.C.Launay, V.Mazoyer, Appl. Phys. Lett. 63 (1993), p.1140.

[38] G.C.Valley, H.Rajbenbach, H.J.Von Bardeleben, Appl. Phys. Lett. 56 (1990), p.364.

[39] M. Kaminska, J.M. Parsey, J. Lagowski, H.C. Gatos, Appl. Phys. Lett. 41 (1982), p.989.

[40] S. Weiss, S. Sternklar, B. Fischer, Opt. Lett. 12 (1987) p.114. 
FIGURE CAPTIONS :

Fig.1 : Energy diagram of semiconductors as a function of a space coordinate. For symbol definition, see text

Fig.2 : Two wave mixing scheme showing energy transfer from the pump beam Ip to the probe beam Is.

Fig.3 : Photorefractive gain $\Gamma$ as a function of the grating wave vector $\mathrm{k}$. The experimental curves show results for $\lambda=1.06 \mu \mathrm{m}(+)$ and for $\lambda=1.32 \mu \mathrm{m}(\times)$. Straight and dashed lines represent theoretical curves.

Fig. 4 : EPR spectrum of the EL2 defect.

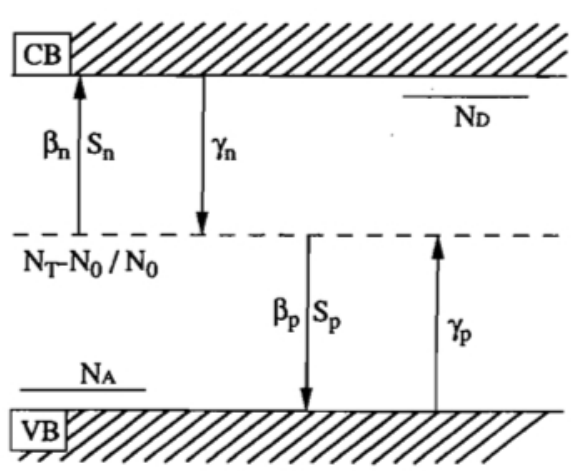

Figure 1

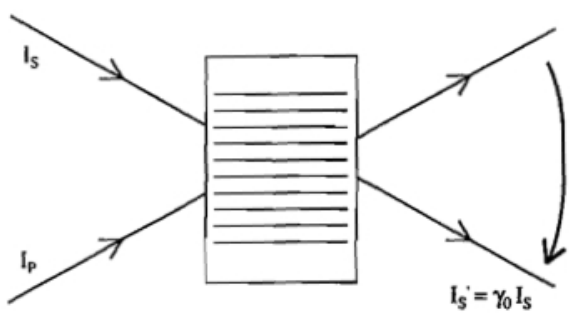

Figure 2 


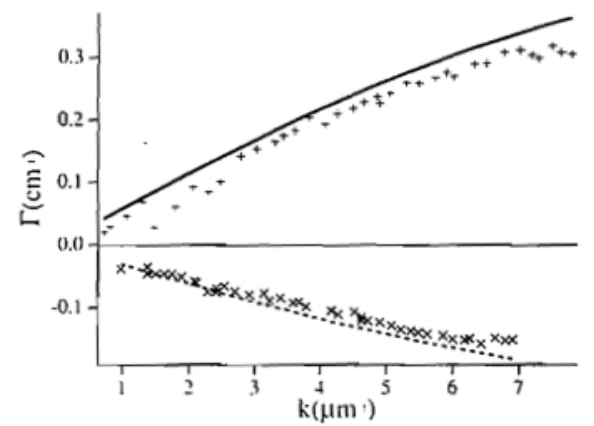

Figure 3

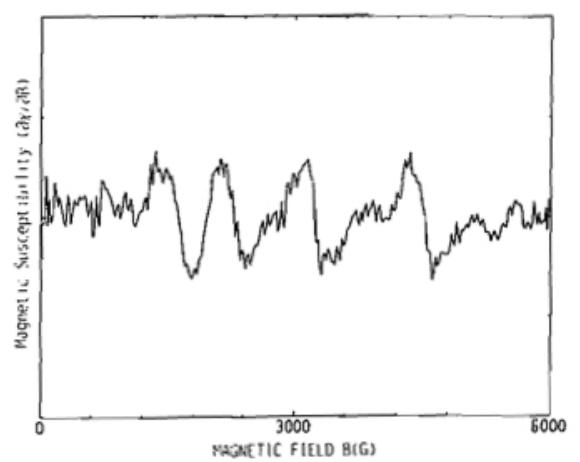

Figure 4 